\title{
Gait in Mild Alzheimer's Disease: Feasibility of Multi-Center Measurement in the Clinic and Home with Body-Worn Sensors: A Pilot Study
}

Ríona Mc Ardle, Rosie Morrisa, Aodhán Hickey, Silvia Del Din, Ivan Koychev, Roger N. Gunn, Jennifer Lawson, Giovanna Zamboni, Basil Ridha, Barbara J. Sahakian, James B. Rowe, Alan Thomas, Henrik Zetterberg, Clare MacKay, Simon Lovestone and Lynn Rochester, on behalf of the Deep and Frequent Phenotyping study team

[Journal of Alzheimer's Disease 63(1), 2018, 331-341, DOI 10.3233/JAD-171116]

https://content.iospress.com/articles/journal-of-alzheimers-disease/jad171116

On page 334, in figure 1(e), the term "micro gait" appears on the left hand side as well as on the right hand side. On the right hand side, however, it should read "macro gait". The correct figure is included below.

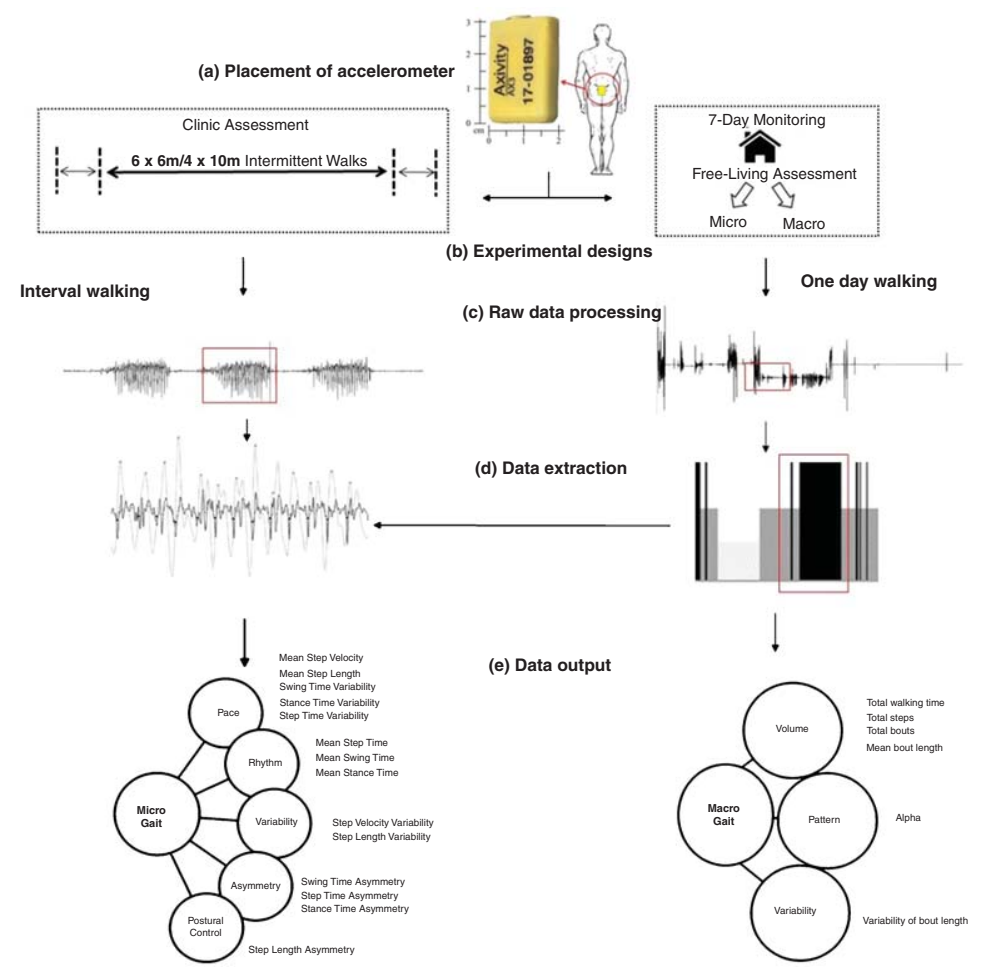

